I dent ificati on and Char act erizati on of Novel Genes Expressed Preferenti al Iy in the Corpora Al I at a or Corpora Cardi aca during the Juveni I e Hor mone Synt het i c Peri od in the Si I kworm, Bonbyx mor i

\begin{tabular}{|l|l|}
\hline 著者 & $\begin{array}{l}\text { Taguchi Syusaku, I wam Nasaf um , K ya } \\
\text { Taket oshi }\end{array}$ \\
\hline 著者別表示 & 岩見 雅史, 木矢 剛智 \\
\hline $\begin{array}{l}\text { j our nal or } \\
\text { publ i cat i on ti t l e }\end{array}$ & Zool ogi cal Sci ence \\
\hline vol une & 34 \\
\hline nunber & 5 \\
\hline page range & 398 405 \\
\hline year & $2017-10$-01 \\
\hline URL & ht t p: //doi . or g/10. 24517/00049521 \\
\hline
\end{tabular}




\section{Identification and Characterization of Novel Genes Expressed Preferentially in the Corpora Allata or Corpora Cardiaca During the Juvenile Hormone Synthetic Period in the Silkworm, Bombyx mori}

Author(s): Syusaku Taguchi, Masafumi Iwami and Taketoshi Kiya

Source: Zoological Science, 34(5):398-405.

Published By: Zoological Society of Japan

https://doi.org/10.2108/zs170069

URL: http://www.bioone.org/doi/full/10.2108/zs170069

BioOne (www.bioone.org) is a nonprofit, online aggregation of core research in the biological, ecological, and environmental sciences. BioOne provides a sustainable online platform for over 170 journals and books published by nonprofit societies, associations, museums, institutions, and presses.

Your use of this PDF, the BioOne Web site, and all posted and associated content indicates your acceptance of BioOne's Terms of Use, available at www.bioone.org/page/terms_of_use.

Usage of BioOne content is strictly limited to personal, educational, and non-commercial use. Commercial inquiries or rights and permissions requests should be directed to the individual publisher as copyright holder. 


\title{
Identification and Characterization of Novel Genes Expressed Preferentially in the Corpora Allata or Corpora Cardiaca During the Juvenile Hormone Synthetic Period in the Silkworm, Bombyx mori
}

\author{
Syusaku Taguchi, Masafumi Iwami, and Taketoshi Kiya* \\ Division of Life Sciences, Graduate School of Natural Science and Technology, \\ Kanazawa University, Kanazawa, Ishikawa, Japan
}

\begin{abstract}
Juvenile hormone $(\mathrm{JH})$ plays important roles in insect development and physiology. JH titer is tightly regulated to coordinately adjust systemic physiology and development. Although control of $\mathrm{JH}$ titer is explained by the expression of $\mathrm{JH}$ biosynthetic enzymes in the corpora allata (CA), molecular mechanisms that regulate the expression of these genes remain elusive. In the present study, to identify novel regulators of JH biosynthetic genes, we conducted a gene expression screen using the CA and corpora cardiaca (CC) of the silkworm, Bombyx mori, in the JH synthesis period. We identified seven candidate genes and characterized their properties through extensive expression analyses. Of these candidates, we found that a novel gene, which encodes type II phosphatidylinositol 3,4-bisphosphate $\left[\mathrm{PI}(3,4) \mathrm{P}_{2}\right]$ 4-phosphatase, shows highly correlated expression with JH titer. In addition, expression of this gene was strongly upregulated by starvation, when JH biosynthetic enzyme genes are concurrently upregulated. These results, for the first time, imply possible involvement of phosphoinositol signal in regulation of $\mathrm{JH}$ biosynthesis, providing novel insights into molecular mechanisms of nutrition-dependent regulation of $\mathrm{JH}$ biosynthesis.
\end{abstract}

Key words: Bombyx mori, JH, corpora allata, phosphoinositol, development

\section{INTRODUCTION}

Postembryonic development in insects is regulated by coordinated actions of ecdysone and juvenile hormone (JH) (Nijhout, 1994). Active metabolite of ecdysone, 20-hydroxyexdysone (20E), triggers molting events, and $\mathrm{JH}$ exerts "status quo" effects on larval development. A rise of $20 \mathrm{E}$ titer promotes larva-to-larva development under a high $\mathrm{JH}$ condition, whereas it leads to larva-to-pupa metamorphosis under a low JH condition (Nijhout, 1994; Riddiford, 1996). In addition to its effects on larval development, JH exerts pleiotropic effects on insect physiology, such as reproduction, diapause, and polyphenism (Denlinger, 2005; Hartfelder, 2005; Raikhel, 2005). These functions of JH titer are tightly regulated by concerted actions of $\mathrm{JH}$ biosynthetic and degradative enzymes (Nijhout, 1994; Tan et al., 2005; Kinjoh et al., 2007).

$\mathrm{JH}$ is synthesized and secreted from the corpora allata $(\mathrm{CA})$, a neurosecretory organ that is connected to the brain through the corpora cardiaca (CC). JH biosynthesis consists of a series of enzymatic reactions that are conventionally divided into two major phases: the early steps where farnesyl pyrophosphate (FPP) is synthesized through the mevalonate pathway and the late steps where FPP is converted to $\mathrm{JH}$. The mevalonate pathway is highly conserved among

\footnotetext{
* Corresponding author. E-mail: kiya@staff.kanazawa-u.ac.jp doi:10.2108/zs170069
}

animals; FPP is synthesized from acetyl-CoA or acetoacetyl-CoA through the actions of eight enzymes, as follows: acetoacetyl-CoA thiolase (AACT), HMG-CoA synthase (HMGS), HMG-CoA reductase (HMGR), mevalonate kinase (MevK), phosphomevalonate kinase (MevPK), diphosphomevalonate decarboxylase (MevPPD), isopentenyl diphosphate isomerase (IPPI), and farnesyl diphosphate synthase (FPPS). The late steps are arthropod specific, where $\mathrm{JH}$ is synthesized from FPP through the actions of five enzymes as follows; FPP pyrophosphatase, farnesol dehydrogenase, farnesal dehydrogenase, farnesoic acid epoxidase, and juvenile hormone acid methyltransferase (JHAMT) (Shinoda and Itoyama, 2003; Kinjoh et al., 2007; Ueda et al., 2009; Daimon et al., 2012). Because JH is immediately secreted from the CA to hemolymph upon biosynthesis and is not stored, JH titer correlates with the levels of expression of $\mathrm{JH}$ biosynthetic enzyme genes (Shinoda and Itoyama, 2003; Kinjoh et al., 2007). Thus, JH biosynthetic activity of CA is controlled by the regulation of expression levels of these enzyme genes.

$\mathrm{JH}$ biosynthesis is significantly affected by nutritional signals. In the larvae of the tobacco hornworm, Manduca sexta, $\mathrm{JH}$ titer is increased by starvation and is subsequently decreased by re-feeding (Cymborowski, 1982). Furthermore, starvation-induced increase in $\mathrm{JH}$ titer inhibits imaginal disc formation, allowing larvae to conduct an extra molt until they reach the critical weight (Truman et al., 2006). These phenomena indicate that nutritional signals and inter- 
nal physiological states converge onto $\mathrm{JH}$ biosynthetic activity of CA to coordinate systemic physiology. However, the molecular mechanisms underlying the effects of nutritional signals on $\mathrm{JH}$ biosynthetic activity remain elusive.

$\mathrm{JH}$ biosynthesis is also modulated by various factors including neuropeptides, such as allatotropin (AT) and short neuropeptide F (sNPF) (Goodman, 2005; Stay and Tobe, 2007; Audsley et al., 2008; Yamanaka et al., 2008; Kaneko and Hiruma, 2014), neurotransmitters, (Granger et al., 1996; Gilbert et al., 2000; Granger et al., 2000), decapentaplegic (Huang et al., 2011), TGF- $\beta$ signaling (Ishimaru et al., 2016), and the molting hormone 20E (Whisenton et al., 1985; Kaneko et al., 2011). A recent transcriptomic study revealed that AT receptor (ATR), which had long been believed to be expressed in the $C A$, is preferentially expressed in the CC, but not in the CA. In addition, sNPF released from ATRpositive CC cells functions as an allatoregulatory peptide, (Yamanaka et al., 2008). These studies indicate that both the $\mathrm{CA}$ and the $\mathrm{CC}$ are involved in $\mathrm{JH}$ biosynthesis.

In the present study, we identified genes preferentially expressed in the $\mathrm{CA}$ or $\mathrm{CC}$ during $\mathrm{JH}$ synthetic period in the larvae of silkworm, B. mori. One of these genes, which encodes type II phosphatidylinositol 3,4-bisphosphate $\left[\mathrm{PI}(3,4) \mathrm{P}_{2}\right]$ 4-phosphatase, showed an expression pattern that is highly correlated with $\mathrm{JH}$ titer and strongly enhanced expression under starvation. These results imply that phosphoinositol signaling pathway may regulate $\mathrm{JH}$ biosynthetic activity of CA in the downstream of nutritional signals.

\section{MATERIALS AND METHODS}

\section{Insects}

Eggs of a racial hybrid of $B$. mori, Kinshu $x$ Showa, were purchased from a local dealer (Ueda Sanshu, Nagano, Japan). Larvae were reared on an artificial diet (Silkmate 2M, Nihon Nosan Kogyo, Yokohama, Japan) at $25^{\circ} \mathrm{C}$ under a $12 \mathrm{~h}$ light $/ 12 \mathrm{~h}$ dark photoperiod. The day of ecdysis was designated as day zero (IV0 or V0).

\section{Fluorescent differential display}

The CAs and CCs (101-106 tissue samples, each) were collected from day four of fourth instar (IV4) or day five of fifth instar (V5) larvae. Total RNA was isolated with TRIzol (Invitrogen, Carlsbad, CA), treated with DNase I, and reverses transcribed (RT) with SuperScript III (Invitrogen). Fluorescent differential display was performed as described previously using a Fluorescent Differential Display Kit and LA Taq polymerase (Takara-Bio, Shiga, Japan) with partial modification (Ito and Sakaki, 1999; Taguchi et al., 2011). To verify the reproducibility of experiments, screening was conducted in duplicate. Bands of interest were excised, reamplified, and subcloned into a pGEM-T vector (Promega, Madison, WI). Three clones for each band were isolated and the sequences of all clones were determined.

\section{Semi-quantitative RT- PCR}

Total RNA was isolated from CAs and CCs (30 tissue samples each) of IV4 or V5 larvae, treated with DNase I, and reverse transcribed with ReverTra Ace (Toyobo, Osaka, Japan). Semiquantitative RT-PCR was performed with GoTaq Green Master Mix (Promega) using gene-specific primers (Table 1), following the manufacturer's protocol. PCR conditions were 33-45 cycles of $94^{\circ} \mathrm{C}$ for $30 \mathrm{~s}, 57^{\circ} \mathrm{C}$ for $30 \mathrm{~s}$, and $72^{\circ} \mathrm{C}$ for $30 \mathrm{~s}$. Cycles of amplification was adjusted to the level of each gene expression, which was estimated by preliminary experiments. Ribosomal protein L3 (rp/3) was used as an internal control. PCR without RT reaction was included and confirmed no genomic contamination. To secure the reproducibility of semi-quantitative $\mathrm{RT}-\mathrm{PCR}$, all experiments were conducted in triplicate.

\section{Real-time RT-PCR}

The precise levels of gene expression were determined by real-time RT-PCR. Total RNA was isolated from CA-CC complexes (20-40 each) or various tissues (3-7 each) for each sample. The total RNA was treated with DNase I, and reverse transcribed with PrimScript RT Master mix (Takara-Bio). Real-time RT-PCR was performed with and SYBR Premix Ex Taq ${ }^{\mathrm{TM}}$ II , using gene-specific primers (Table 2). Levels of JH biosynthetic enzyme gene expression were determined using the gene-specific primers described previously (Shinoda and Itoyama, 2003; Kinjoh et al., 2007; Daimon et al., 2012). PCR condition was $95^{\circ} \mathrm{C}$ for $30 \mathrm{~s}$, followed by 40 cycles of $95^{\circ} \mathrm{C}$ for $5 \mathrm{~s}$ and $60^{\circ} \mathrm{C}$ for $30 \mathrm{~s}$. The fluorescent signals from samples (SYBR Green) were obtained using ABI7900HT (Applied Biosystems, Foster City, CA) with default settings. The level of gene

Table 1. Sequence of primers used for semi-quantitative RT-PCR.

\begin{tabular}{ll}
\hline \multicolumn{1}{c}{ Primers } & \multicolumn{1}{c}{ Sequences (5' to 3') } \\
\hline BGIBMGA007861/Forward & AGTCGATGGAGTAGCGTTCTTTG \\
BGIBMGA007861/Reverse & GGCATCGTCCTTCAGTTCTTG \\
Nene/Forward & TCTGCGATCCTGTCCAGCCA \\
Nene/Reverse & ACAGCTCACCGCCTACCCGA \\
BGIBMGA005735/Forward & ATTGGCGCCGCTAGGCTTGG \\
BGIBMGA005735/Reverse & CCGTCGGCGAGAACCAGCTC \\
BGIBMGA002123/Forward & GGGCCAGCGACGGTCTGAAC \\
BGIBMGA002123/Reverse & AAAGGGACACGCGCAGCCTC \\
BGIBMGA008114/Forward & TACGGCGACGGAAAGGTGCG \\
BGIBMGA008114/Reverse & GGGAGAGGTTTCTCGCACGGG \\
BGIBMGA012888/Forward & AGCTCGCTATTCGCGCCCAC \\
BGIBMGA012888/Reverse & CGACGAGCCAGGTGCCGAAG \\
BGIBMGA007603/Forward & GAAGGCCGAACACCCTGGCG \\
BGIBMGA007603/Reverse & GCTCGTTGGTCCACTGCGGG \\
RpL3/Forward & AGCACCCCGTCATGGGTCTA \\
RpL3/Reverse & TGCTCCAAGCTCATCCTGC \\
\hline
\end{tabular}

Table 2. Sequence of primers used for real-time RT-PCR.

\begin{tabular}{ll}
\hline \multicolumn{1}{c}{ Primers } & \multicolumn{1}{c}{ Sequences (5' to 3') } \\
\hline BGIBMGA007861/Forward & AGTCGATGGAGTAGCGTTCTTTG \\
BGIBMGA007861/Reverse & GGCATCGTCCTTCAGTTCTTG \\
Nene/Forward & TCTGCGATCCTGTCCAGCCA \\
Nene/Reverse & ACAGCTCACCGCCTACCCGA \\
BGIBMGA005735/Forward & AAGCCGAACCCTCACCAA \\
BGIBMGA005735/Reverse & TGCCGACTTCATCCACCA \\
BGIBMGA002123/Forward & GGGCCAGCGACGGTCTGAAC \\
BGIBMGA002123/Reverse & AAAGGGACACGCGCAGCCTC \\
BGIBMGA008114/Forward & ACTCGCAACCAGCATCTCC \\
BGIBMGA008114/Reverse & TCGGGAATAGGTGTGGATAAAAC \\
BGIBMGA012888/Forward & GCCAAGGTCTTCGTCCAATC \\
BGIBMGA012888/Reverse & TTGCTGTTTCGTAGATGCTGTTC \\
BGIBMGA007603/Forward & GAAGGCCGAACACCCTGGCG \\
BGIBMGA007603/Reverse & GCTCGTTGGTCCACTGCGGG \\
RpL3/Forward & AAGAGATCGTGGAGGCTGTCA \\
RpL3/Reverse & CGTAGTCCATGAGGGGTCTCA \\
\hline
\end{tabular}


A

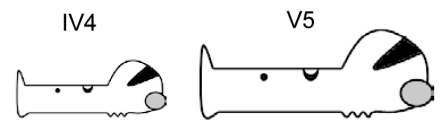

High JH synthesis

Low JH synthesis

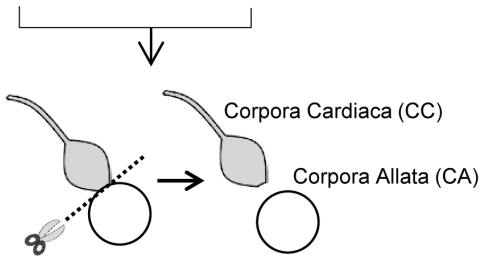

B

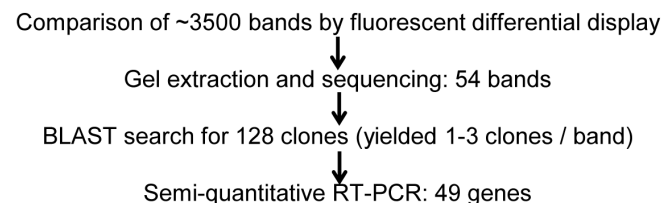

C
Gene name

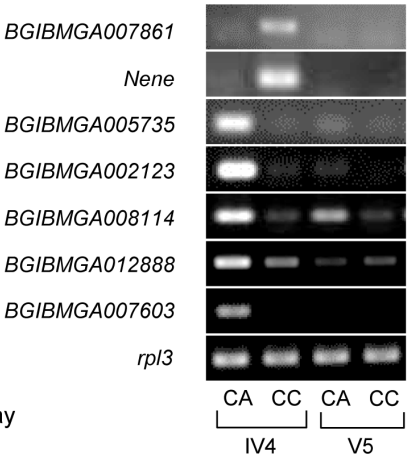

Homologous gene

Type XI Collagen $\alpha-1$

Non-coding RNA

Desmosterol reductase

Type II PI $(3,4) P_{2}$ 4-phosphatase

GUK-holder

Steroid dehydrogenase

Cytochrome B5
Fig. 1. Screening of genes expressed preferentially during the JH synthesizing stage in the CA and CC. (A) Schematic illustration of the screening procedures. The CA-CC complex was dissected from IV4 (high JH titer) or V5 (low JH titer) larvae. The CA and CC were separated and used for fluorescent differential display. (B) Flowchart of screening. (C) Results of semi-quantitative RT-PCR. Seven genes were confirmed to be expressed in a JH synthesizing stage-selective manner in the CA and CC. Gene names and their homologues were listed in the left and right side of band pictures, respectively. Experiments were conducted in triplicate (biological replicates). The bands of $r p / 3$ showed similar intensity among samples, indicating that similar amounts of RNA were used. PCR without RT reaction confirmed no genome contamination (not shown). expression was determined using the serially diluted (10-fold dilutions for a dynamic range of $\left.10^{6}\right)$ quantification standards, which was prepared from purified PCR products. The determined value of each sample was normalized to that of $r p / 3$. The normalized values were again normalized to that of control sample, and shown as the relative expression. Statistical analyses were conducted by the $F$-test and Student's $t$-test, using Microsoft Excel (Microsoft). All data are presented as mean \pm standard error.

To identify the full-length cDNA sequence of candidate genes, $5^{\prime}$ - and $3^{\prime}$-RACE were performed repeatedly using the CA-CC complex total RNA and the SMART RACE cDNA Amplification Kit (Clontech, Mountain View, CA). The cDNA sequences were analyzed using Geneious Pro 7 (Biomatters, Auckland, New Zealand).

\section{Whole-mount in situ hybridization}

The digoxigenin (DIG)-labeled RNA probes were synthesized using a DIG RNA labeling mix (Roche). Template DNA fragments for RNA probes were obtained using gene-specific primers [BGIBMGA007861; 5'-CCAGGCAGACAAGGACGCCG-3' and 5'-CGATTCGCCCGTGGCACCTT-3', BGIBMGA005735; 5'-ATTGGCGCCGCTAGGCTTGG-3' and 5'-TTAGGCAGCGAAGCCCGCAC-3', BGIBMGA002123; 5'-GGGCCAGCGACGGTCTGAAC-3' and 5'-AAAGGGACACGCGCAGCCTC-3', BGIBMGA012888; 5'-AAGCTGTTAGCCGCCCGTGG-3' and 5'-CGACGAGCCAGGTGCCGAAG-3']. The PCR products were subcloned into a pGEM-T vector (Promega) and insert sequences were confirmed.

Whole-mount in situ hybridization was conducted as described previously (Ueda et al., 2009). CA-CCbrain complexes were dissected from IV4 or V5 larvae, fixed in $4 \%$ formaldehyde in phosphate-buffered saline with $0.1 \%$ Tween 20 (PBTw) overnight at $4^{\circ} \mathrm{C}$, gradually

\section{Rapid amplification of cDNA ends (RACE)}

A

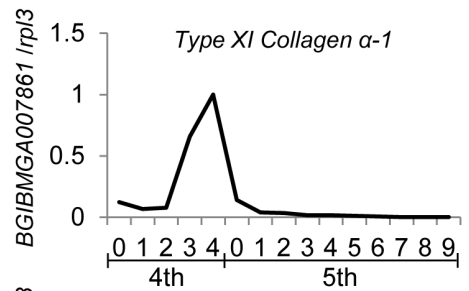

C

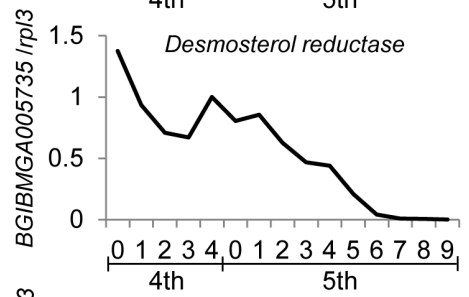

E

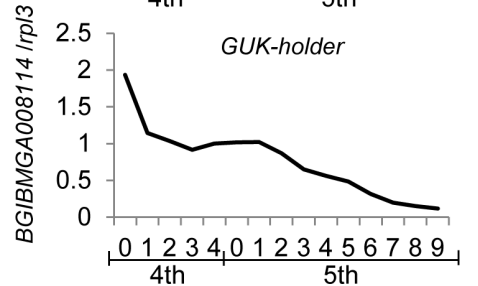

G

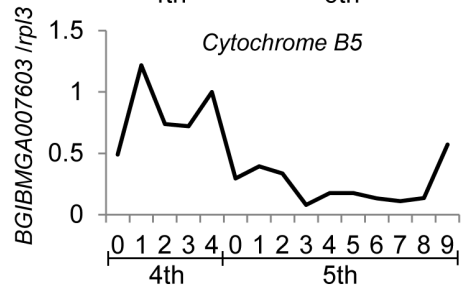

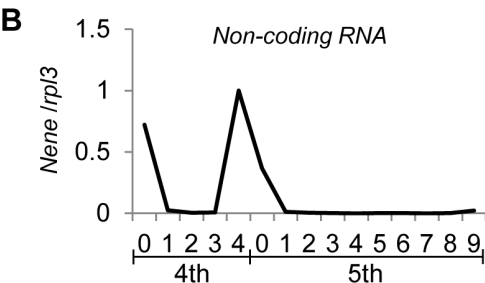

D
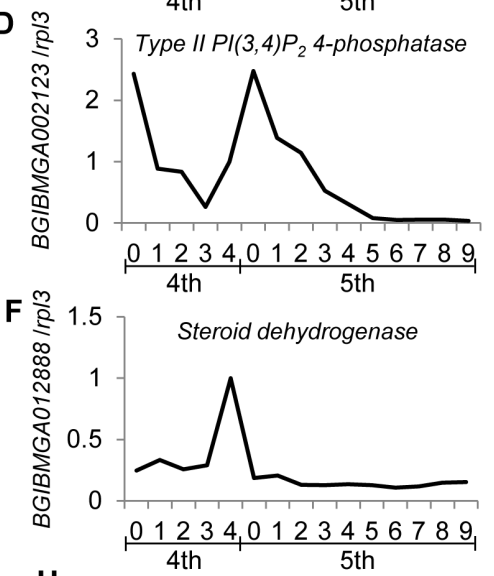

H

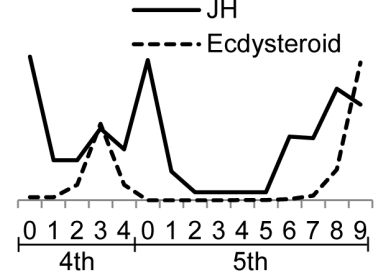

Fig. 2. Temporal expression profiles of candidate genes in the CA-CC complexes of fourth and fifth-instar larvae. (A-G) The levels of candidate gene expression were quantified by real-time RT-PCR. Relative values to IV4 larvae are shown. One sample (prepared from 20-40 CA-CC complexes) per stage was analyzed. (H) Temporal titer changes in the $\mathrm{JH}$ and ecdysteroid in the silkworm. The $\mathrm{JH}$ and ecdysteroid titer are depicted according to previous papers (Sakurai and Niimi, 1997; Satake et al., 1998; Koyama et al., 2004). 
dehydrated with serial concentrations of ethanol/PBTw, and stored in $100 \%$ ethanol at $-30^{\circ} \mathrm{C}$ until use. On the day of hybridization, the samples were gradually rehydrated with serial concentrations of ethanol/PBTw, permeabilized with proteinase $\mathrm{K}(5 \mu \mathrm{g} / \mathrm{ml})$ in PBTw for $75 \mathrm{sec}$ at room temperature, washed with PBTw, refixed in $4 \%$ formaldehyde in PBTw for 30 min, washed with PBTw, incubated in prehybridization solution $(50 \%$ formamide, $5 \times$ saline sodium citrate [SSC] [pH 7.0], 2\% Blocking reagent [Roche], 0.1\% TritonX100, $0.1 \%$ CHAPS, $1 \mu \mathrm{g} / \mathrm{ml}$ yeast tRNA, $5 \mathrm{mM}$ EDTA, and $5 \mu \mathrm{g} / \mathrm{ml}$ heparin) for $1 \mathrm{~h}$ at $50^{\circ} \mathrm{C}$, and hybridized with RNA probes $(1 \mu \mathrm{g} / \mathrm{ml})$ overnight at $50^{\circ} \mathrm{C}$ in prehybridization solution. After washes in SSCFS $(50 \%$ formamide, $5 \times \mathrm{SSC}[\mathrm{pH} 4.5]$, and $1 \% \mathrm{SDS}$ ) for $20 \mathrm{~min}$ at $50^{\circ} \mathrm{C}$, the samples were washed three times in $2 \times \operatorname{SSCC}(2 \times \mathrm{SSC}$ [pH 7.0], $0.1 \%$ CHAPS) for $20 \mathrm{~min}$ at $50^{\circ} \mathrm{C}$, three times in $0.2 \times \operatorname{SSCC}(0.2 \times$ SSC [pH7.0], $0.1 \%$ CHAPS) for $20 \mathrm{~min}$ at $50^{\circ} \mathrm{C}$, and three times in KTBT $(50 \mathrm{mM}$ Tris- $\mathrm{HCl}$ [pH7.5], $150 \mathrm{mM} \mathrm{NaCl}, 10 \mathrm{mM} \mathrm{KCl}, 0.1 \%$ Triton $\mathrm{X}-100$ ) for $10 \mathrm{~min}$ at room temperature, then treated with blocking solution (1.5\% Blocking reagent [Roche] in KTBT) for $1 \mathrm{~h}$, and then incubated in alkaline phosphatase-conjugated anti-DIG antibody $\left(1 / 2500\right.$, Roche) in blocking solution overnight at $4^{\circ} \mathrm{C}$. After washes in KTBT for $1 \mathrm{~h}$ five times at room temperature and washes in NTMT $(100 \mathrm{mM} \mathrm{NaCl}, 100 \mathrm{mM}$ Tris- $\mathrm{HCl}$ [pH 9.5], $50 \mathrm{mM} \mathrm{MgCl} 2,0.1 \%$ Triton $\mathrm{X}-100)$ for $5 \mathrm{~min}$ three times, signals were developed with NBT/BCIP solution (1/50 dilution in NTMT, Roche) for $1 \mathrm{~h}$ or overnight at room temperature with continuous agitation. The colordeveloped samples were washed in PBTw, fixed in 4\% formaldehyde/ PBTw, washed in PBTw, washed in serial concentrations of ethanol/PBTw, and mounted in 50\% glycerol/PBTw. Images were obtained using the BZ-9000 microscope (Keyence, Osaka, Japan).

\section{Hormone treatments}

Fenoxycarb (Wako, Osaka, Japan), dissolved in acetone $(1 \mathrm{mg} / \mathrm{ml})$, was applied topically to IV1 larvae $(5 \mu \mathrm{I}$ each). 20E (Sigma, St. Louis, MO), dissolved in distilled water $(1 \mathrm{mg} / \mathrm{ml})$, was injected into IV1 larvae $(3 \mu \mathrm{l})$. For control experiments, the same amount of solvent was topically applied or injected.

\section{RESULTS}

Identification of genes preferentially expressed in the CA or CC during $\mathrm{JH}$ biosynthetic stage

To identify novel genes that regulate $\mathrm{JH}$ biosynthesis, we conducted a fluorescent differential display screening using the $\mathrm{CA}$ and CC of silkworm larvae (Fig. $1 \mathrm{~A})$. We compared gene expression patterns between day four of fourth instar (IV4) and day five of fifth instar (V5) larvae, when $\mathrm{JH}$ biosynthesis is active and inactive, respectively (Sakurai and Niimi, 1997; Kinjoh et al., 2007). In addistage/tissue was analyzed. tion, we separately compared gene expression patterns between $\mathrm{CA}$ and $\mathrm{CC}$ on these stages. We compared approximately 3500 bands and identified 49 candidate genes. 26, eight, eight, and seven candidates were identified from IV4 CA, IV4 CC, V5 CA, and V5 CC, respectively (Fig. 1B). AACT and JHAMT, known as JH biosynthetic enzyme genes and expressed specifically in the $\mathrm{CA}$ of $\mathrm{JH}$ biosynthetic stage, were included in these candidates, confirming that our screening was conducted appropriately (Shinoda and Itoyama, 2003; Kinjoh et al., 2007). After further verification by semi-quantitative RT-PCR, database search using the silkworm genome information, and determination of transcript sequences by RACE, we selected seven novel genes for further analyses; five and two genes expressed strongly in IV4 CA and IV4 CC, respectively (Fig. 1C). Since no homologous gene or clear open reading frame was identified for one of the IV4 CC-expressed genes, we named this gene nene (noncoding RNA expressed in the nervous sys-

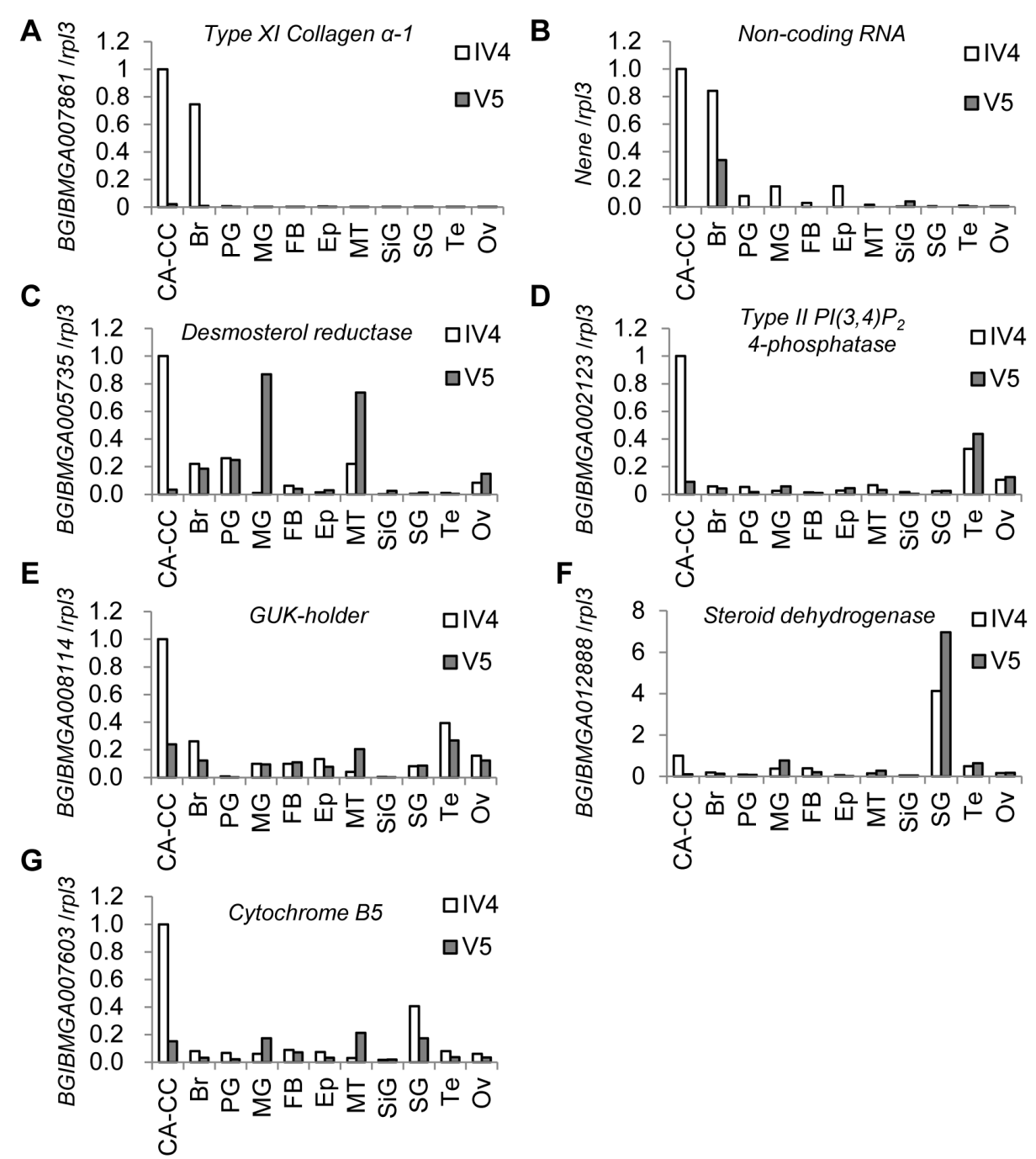

Fig. 3. Tissue expression profiles of candidate genes in the IV4 and V5 larvae. (A-G) The levels of identified gene expression were precisely quantified by real-time RT-PCR. Relative values to CA-CC complex of IV4 larvae are shown. Br, brain; PG, prothoracic gland; MG, midgut; FB, fat body; Ep, epidermis; MT, Malpighian tubule; SiG, silk gland; SG, salivary gland; Te, testis; Ov, ovary. One sample (prepared from 20-40 CA-CC complexes or three to seven tissues) per 
tem at ecdysis: Accession \# LC222462).

\section{Temporal expression profiles of candidate genes}

Expression levels of known $\mathrm{JH}$ biosynthetic enzyme genes correlate with $\mathrm{JH}$ titer and show dynamic changes throughout development. In order to investigate whether the newly identified candidate genes show similar patterns in expression levels, we investigated temporal expression profiles of the candidate genes from IV0 to V9 with real-time RT-PCR using the CA-CC complexes (Fig. 2). BGIBMGA007861 (Type XI Collagen $\alpha-1$ ), nene, BGIBMGA005735 (Desmosterol reductase), BGIBMGA002123 (Type I/ PI(3,4)P $P_{2}$ 4-phosphatase), and BGIBMGA012888 (Steroid dehydrogenase) showed correlated expression profile with $\mathrm{JH}$ titer until the first half of fifth instar. Especially, the expression of BGIBMGA002123 highly correlated with $\mathrm{JH}$ titer, peaking in $\mathrm{V} 0$ instar. In contrast, expression levels of these genes did not correlate with the rise of $\mathrm{JH}$ titer in the latter half of fifth instar. Since increase in $\mathrm{JH}$ titer in late fifth instar is derived from the gonad and not the CA (Sakurai and Niimi, 1997; Kinjoh et al., 2007), the low expression of candidate genes can be reflecting the low $\mathrm{JH}$ production in the CA. These results suggest that these genes are not upregulated in response to increase in $\mathrm{JH}$ titer, but functions upstream or parallel of the $\mathrm{JH}$ biosynthesis pathway in the CA-CC complex. BGIBMGA008114 (GUK-holder) and BGIBMGA007603 (Cytochrome B5) did not show correlated expression profile with $\mathrm{JH}$ titer, suggesting that these genes are not related to $\mathrm{JH}$ biosynthesis.

\section{Tissue expression profiles of candi- date genes}

Next, we performed quantitative RTPCR to reveal tissue expression profiles of the candidate genes (Fig. 3). BGIBMGA007861 (Type XI Collagen $\alpha-1)$ and nene were preferentially expressed in the nervous tissues of IV4 larvae. BGIBMGA002123 (Type II PI(3,4)P 2 4-phosphatase), BGIBMGA008114 (GUK-holder) and BGIBMGA007603 (Cytochrome B5) were preferentially expressed in the CA-CC complex of IV4 larvae. In contrast, BGIBMGA005735 (Desmosterol reductase) and BGIBMGA012888 (Steroid dehydrogenase) were highly expressed in tissues other than the CA-CC complex.

To further confirm the tissue-preferential expression, we conducted whole mount in situ hybridization using the CA-CC complex of IV4 and V5 larvae (Fig. 4). Consistent with the results of RT-PCR, signals of BGIBMGA007861 (Type $X I$ Collagen $\alpha-1$ ) were preferen- tially detected in the CC of IV4 larvae (Fig. 4A). In addition, BGIBMGA005735 (Desmosterol reductase), BGIBMGA002123 (Type II PI(3,4)P 2 4-phosphatase), and BGIBMGA012888 (Steroid dehydrogenase) were preferentially expressed in the CA of IV4 larvae (Fig. 4B-D). In contrast, signals were not detected for nene, BGIBMGA008114 (GUK-holder), and BGIBMGA007603 (Cytochrome B5), possibly due to low abundance of transcripts. These results collectively indicate that expression of BGIBMGA007861 (Type XI Collagen $\alpha-1$ ) and BGIBMGA002123 (Type II $P I(3,4) P_{2}$ 4-phosphatase) spatiotemporally correlate with $\mathrm{JH}$ biosynthesis, implying a possible involvement of these genes in $\mathrm{JH}$ production and/or regulation.

\section{Response of candidate genes to $\mathrm{JH}$ or $20 \mathrm{E}$ treatment}

We next investigated the possibility that correlated expression of candidate genes with $\mathrm{JH}$ titer is not due to their induction by $\mathrm{JH}$. Topical application of a $\mathrm{JH}$ analog, fenoxycarb, to IV1 larvae had no effect on expression levels of candidate genes in the CA-CC complexes $24 \mathrm{~h}$ after treatment (Fig. 5A), indicating that expression of these genes are not regulated by $\mathrm{JH}$. In addition, we investigated

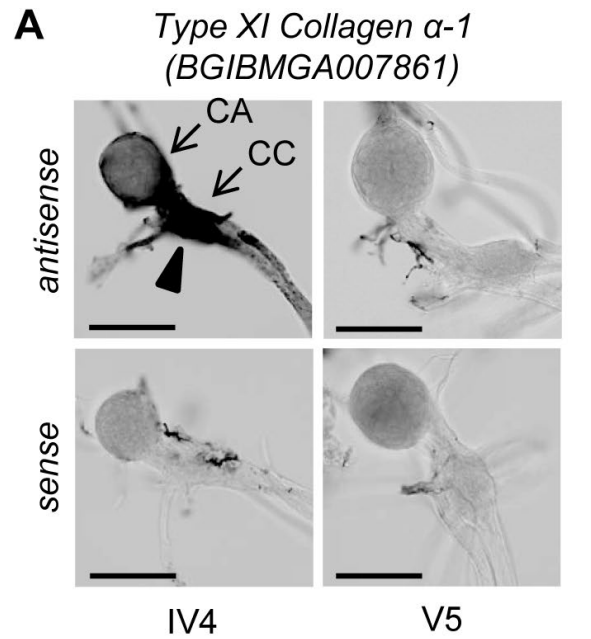

\section{B Desmosterol reductase (BGIBMGA005735)}

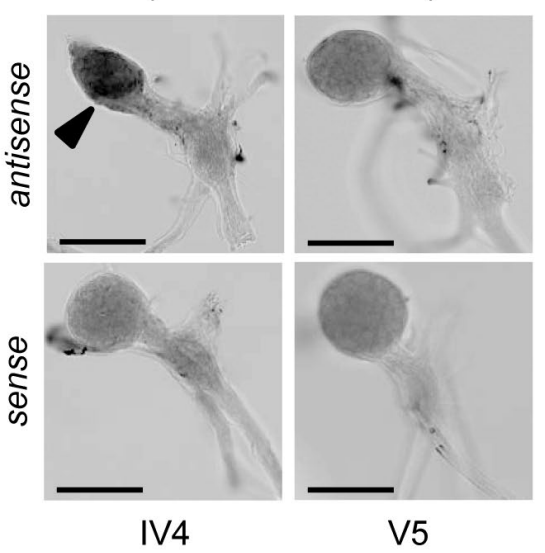

C Type II PI(3,4)P $P_{2}$ 4-phosphatase
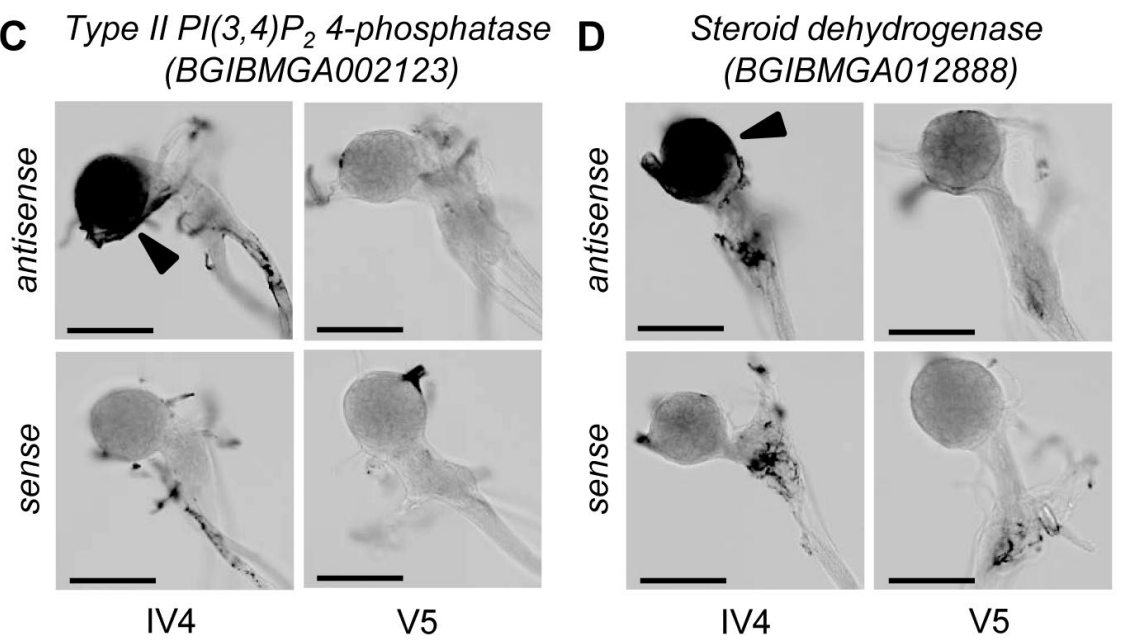

Fig. 4. Whole-mount in situ hybridization of candidate genes in the CA-CC complexes of IV4 and V5 larvae. (A-D) Upper and lower panels indicate results using antisense and sense probes, respectively. Left and right panels are the CA-CC complexes of IV4 and V5 larvae, respectively. Arrowheads indicate stained tissues. Scale bars: $100 \mu \mathrm{m}$. 
the effects of $20 \mathrm{E}$ treatment, to examine if the developmental changes in gene expression levels are due to the dynamic change in ecdysteroid titer from IV3 to IV4. Expression levels of BGIBMGA007861 (Type XI Collagen $\alpha-1$ ) and BGIBMGA012888 (Steroid dehydrogenase) in the CA-CC complexes were upregulated $24 \mathrm{~h}$ after $20 \mathrm{E}$ injection, but other candidate genes were not (Fig. 5B). These results collectively indicate that only BGIBMGA002123 (Type II PI(3,4) $\mathrm{P}_{2}$ 4-phosphatase) remains as the most suitable candidate for upstream regulators of $\mathrm{JH}$ biosynthesis. We did not detect expression of nene in these analyses because of its lack of expression in the CA-CC complexes of IV1 larvae.

\section{Starvation upregulates expression of BGIBMGA002123 (Type II $\mathrm{PI}(3,4) \mathrm{P}_{2}$ 4-phosphatase) and JH biosynthetic genes \\ Nutritional conditions influence JH biosynthesis. To}

A

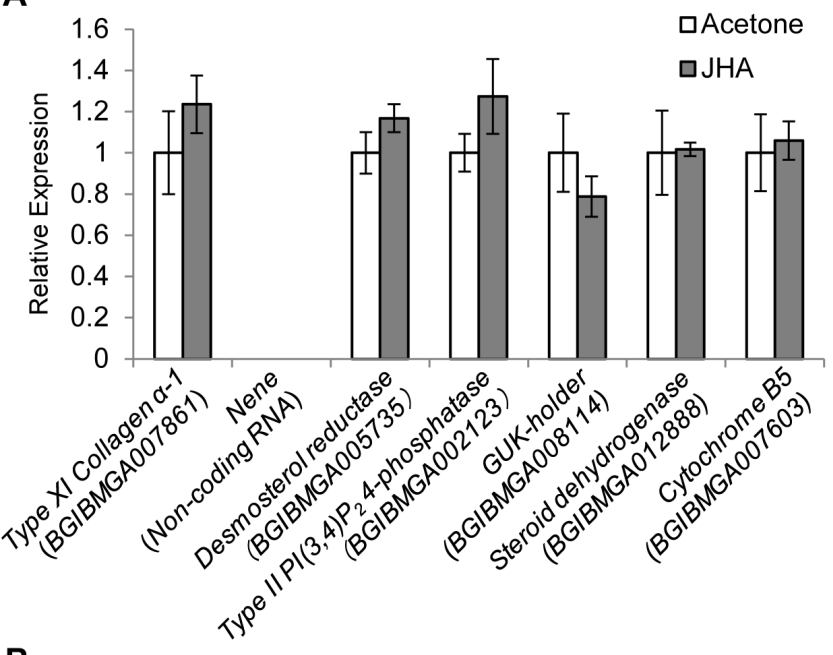

B

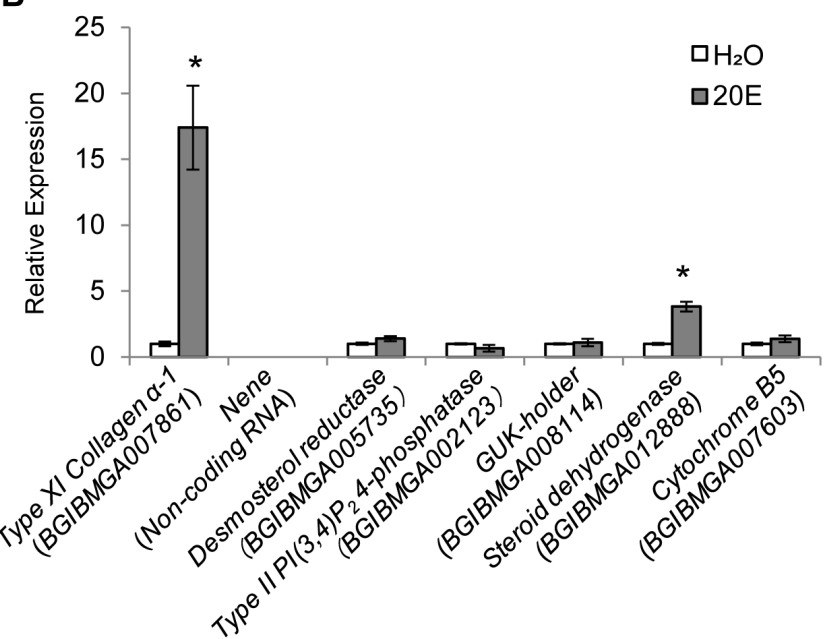

Fig. 5. Effects of hormone treatment on gene expression. Effects of fenoxycarb (JHA) (A) or 20-hydroxyecdysone (20E) (B) treatment on gene expression. The IV1 larvae were treated with JHA (5 $\mu \mathrm{g})(\mathbf{A})$ or $20 \mathrm{E}(3 \mu \mathrm{g})(\mathbf{B})$, and the levels of gene expression in the $\mathrm{CA}-\mathrm{CC}$ complexes were measured $24 \mathrm{~h}$ after treatment. Relative values to control treatment $\left((\mathbf{A})\right.$ acetone, (B) $\left.\mathrm{H}_{2} \mathrm{O}\right)$ are shown. Experiments were conducted in triplicate (biological replicates). *: $P<0.05$, Student's $t$-test. explore possible connections between nutritional conditions and the expression of candidate genes, we examined whether the levels of candidate genes are affected by starvation. Here, we used fourth instar larvae, as the CA of this stage possess $\mathrm{JH}$ synthetic activity and is suitable for assessment of whether starvation further promotes $\mathrm{JH}$ biosynthesis. Levels of candidate gene expression in the CA-CC complexes of larvae starved for two days (from IV1 to IV3) were compared with those of control (fed larvae). Expression of BGIBMGA005735 (Desmosterol reductase) and BGIBMGA002123 (Type /I PI(3,4)P $P_{2}$ 4-phosphatase) was significantly increased in the starved larvae (Fig. 6A). Notably, expression of BGIBMGA002123 (Type II PI(3,4) $P_{2}$ 4-phosphatase) was highly upregulated by starvation. These results suggest that starvation strongly affects phosphoinositol signaling in the $\mathrm{CA}$, since $\mathrm{PI}(3,4) \mathrm{P}_{2}$ 4-phosphatase is the only pathway to metabolize $\mathrm{PI}(3,4) \mathrm{P}_{2}$ to form PI3P and play important roles in phosphoinositol metabolisms (Hawkins and Stephens, 2016). We also investigated levels of $\mathrm{JH}$ biosynthetic enzyme genes in these samples, to examine whether $\mathrm{JH}$ biosynthesis is influenced by starvation (Fig. 6B). Although the levels of HMGCoA synthase (HMGS) and farnesyl acid epoxidase (CYP15C1) expression were significantly decreased by starvation, expression of other enzyme genes were upregulated or unchanged, suggesting that the $\mathrm{JH}$ biosynthesis is prone to upregulation by starvation. These results suggest the possibility that starvation affects phosphoinositol signaling in the $\mathrm{CA}$, which results in upregulation of $\mathrm{JH}$ biosynthesis.

\section{DISCUSSION}

In the present study, we conducted a screen to identify novel regulators of $\mathrm{JH}$ biosynthesis using silkworm larval CA and $\mathrm{CC}$. Through a detailed expression profiling of candidate genes, we identified several novel genes that are preferentially expressed in the CA or $\mathrm{CC}$ of $\mathrm{JH}$ biosynthetic period. Interestingly, expression of BGIBMGA002123, which encodes type II $\mathrm{PI}(3,4) \mathrm{P}_{2}$ 4-phosphatase, showed high correlation with $\mathrm{JH}$ titer and was highly upregulated by starvation, when JH biosynthetic enzyme genes are concurrently upregulated. Although functional analysis using knockout or overexpression animals is necessary, these results imply a novel signaling pathway, where phosphoinositol signaling regulates $\mathrm{JH}$ biosynthesis in the $\mathrm{CA}$.

$\mathrm{JH}$ exerts pleiotropic effects on insect development and physiology (Nijhout, 1994). Therefore, JH titer is tightly controlled by both synthesis and degradation. Although functional analyses of genes for JH biosynthesis and degradation are intensively performed (Shinoda and Itoyama, 2003; Tan et al., 2005; Kinjoh et al., 2007; Niwa et al., 2008; Ueda et al., 2009; Daimon et al., 2012; Daimon et al., 2015), it is still obscure how the expression of $\mathrm{JH}$ biosynthetic enzyme genes in the $\mathrm{CA}$ is regulated to accommodate developmental and physiological demands. Developmental fluctuation of $\mathrm{JH}$ titer is attributable to dynamic changes in expression of $\mathrm{JH}$ biosynthetic enzyme genes (Kinjoh et al., 2007). In the present study, we identified several genes whose expression correlate with $\mathrm{JH}$ titer and characterized their response to hormones, $20 \mathrm{E}$ and $\mathrm{JH}$, and starvation. Our results provide for the first time insights into possible 


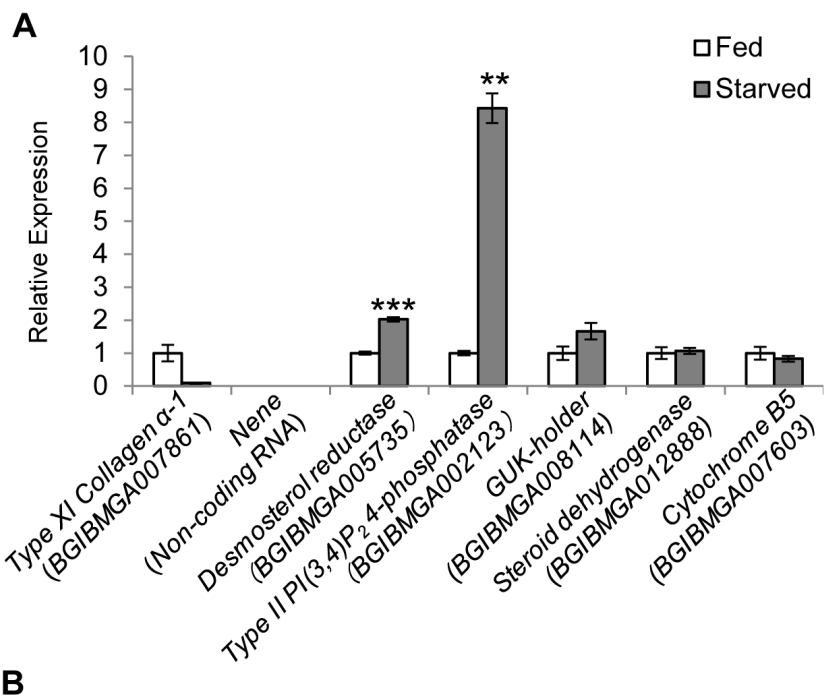

B

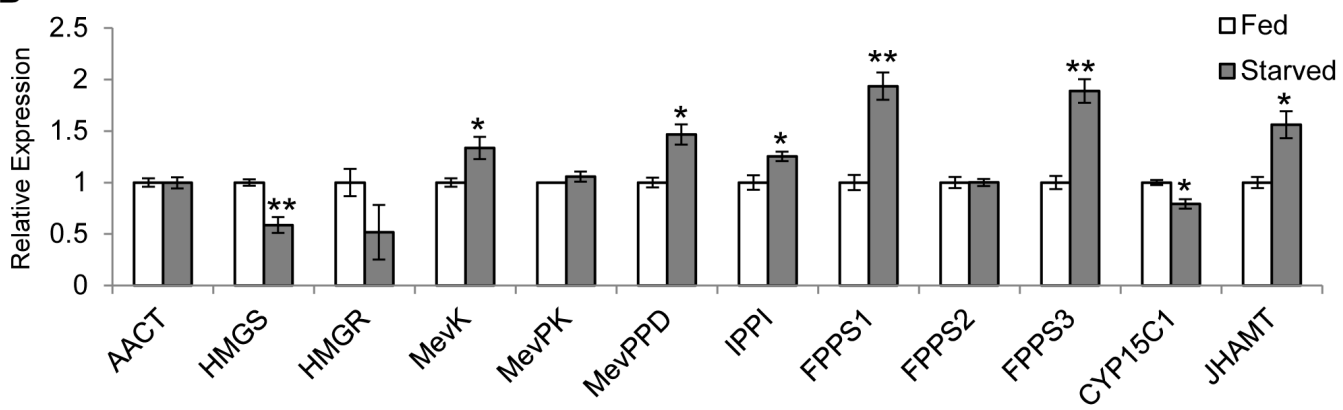

Fig. 6. Starvation regulates gene expression. Effects of starvation on expression levels of candidate genes (A) and $\mathrm{JH}$ biosynthetic enzyme genes (B). (A) IV1larvae were starved for 48 hours, and levels of gene expressions in the CA-CC complexes were measured with real-time RT-PCR. (B) The levels of JH biosynthetic enzyme expression in the CA-CC complexes of starved larvae. AACT, acetoacetyl-CoA thiolase; HMGS, HMG-CoA synthase; HMGR, HMG-CoA reductase; MevK, mevalonate kinase; MevPK, phosphomevalonate kinase; MevPPD, diphosphomevalonate decarboxylase; IPPI, isopentenyl diphosphate isomerase; FPPS, farnesyl diphosphate synthase; CYP15C1, farnesyl acid epoxidase; JHAMT, JH acid $O$-methyltransferase. Experiments were conducted in triplicate (biological replicates). ${ }^{*}: P<0.05,{ }^{* *}: P<$ $0.01,{ }^{* * *}: P<0.001$, Student's $t$-test.

upstream regulators of $\mathrm{JH}$ biosynthesis in the silkworm, $B$. mori.

In some insects, number of larval molting is plastically regulated (Nijhout, 1994). For example, in the last instar larvae of $M$. sexta, additional larval molting is induced when the larval body weight is below the appropriate level for pupation, which is known as the critical weight. Lack of nutrition is sensed by the larval body and induces $\mathrm{JH}$ titer increase, which leads to inhibition of pupal molting and imaginal disc formation (Cymborowski, 1982; Truman et al., 2006). This coordination of systemic physiology and developmental process provides larvae with another chance of pupation under a more appropriate condition. Although this nutrition-dependent regulation of development is a wellknown phenomenon, molecular mechanisms controlling the upregulation of $\mathrm{JH}$ biosynthesis in the CA, is unknown. Therefore, ouridentification of type II PI $(3,4) \mathrm{P}_{2}$ 4-phosphatase as a possible candidate for nutrition-dependent $\mathrm{JH}$ regulator provides insights into molecular mechanisms where systemic physiology adjusts insect development.

How can phosphoinositol signaling regulate $\mathrm{JH}$ biosynthesis in the CA? Type II PI(3,4) $\mathrm{P}_{2}$ 4-phosphatase has $\mathrm{PH}$ overexpressed in the CA or mutated.

(pleckstrin homology) domain and is the major pathway to dephosphorylate $\mathrm{PI}(3,4) \mathrm{P}_{2}$ to form $\mathrm{PI3P}$ in the plasma membrane. Increase in type II $\mathrm{PI}(3,4) \mathrm{P}_{2}$ 4-phosphatase expression is thus expected to decrease the level of $\mathrm{PI}(3,4) \mathrm{P}_{2}$, and increase that of PI3P, respectively. One possible target of $\mathrm{PI}(3,4) \mathrm{P}_{2}$ is Akt/PI3K pathways (Hawkins and Stephens, 2016), which regulates FOXO (forkhead box O) and TOR (target of rapamycin) pathways. Since FOXO and TOR pathways regulate $\mathrm{JH}$ biosynthesis in the adult German cockroach $B$. germanica (Maestro et al., 2009; Suren-Castillo et al., 2012), we speculate that nutrition-dependent change in phosphoinositol signaling may regulate $\mathrm{JH}$ biosynthesis through FOXO and TOR pathway in the CA. In future studies, it will be essential to address this hypothesis using transgenic or knockout silkworms, in which BGIBMGA002123 is

\section{ACKNOWLEDGMENTS}

This work was supported by JSPS KAKENHI Grant Number 12J06522 (S.T.), 24780047 (T.K), and 22380034 (M.I.). We thank S. Takayanagi-Kiya for comments on the manuscript.

\section{COMPETING INTERESTS}

The authors have no competing interests to declare.

\section{AUTHOR CONTRIBUTIONS}

ST performed all experiments. ST and TK designed and wrote the paper. Ml advised on the study.

\section{REFERENCES}

Audsley N, Matthews HJ, Price NR, Weaver RJ (2008) Allatoregulatory peptides in Lepidoptera, structures, distribution and functions. J Insect Physiol 54: 969-980

Cymborowski B, Bogus M, Beckage NE, Williams CM, Riddiford LM (1982) Juvenile hormone titers and metabolism during starvation-induced supernumerary larval moulting of the tobacco hornworm, Manduca sexta L. J Insect Physiol 28: 129-135

Daimon T, Uchibori M, Nakao H, Sezutsu H, Shinoda T (2015) 
Knockout silkworms reveal a dispensable role for juvenile hormones in holometabolous life cycle. Proc Natl Acad Sci USA 112: E4226-4235

Daimon T, Kozaki T, Niwa R, Kobayashi I, Furuta K, Namiki T, et al. (2012) Precocious metamorphosis in the juvenile hormonedeficient mutant of the silkworm, Bombyx mori. PLoS Genet 8: e1002486

Denlinger DL, Yocum GD, Rinehart JP (2005) Hormonal control of diapause. In "Comprehensive Molecular Insect Science" Ed by LI Gilbert, K latrou, SS Gill, Elsevier Ltd., Oxford, pp 615-650

Gilbert LI, Granger NA, Roe RM (2000) The juvenile hormones: historical facts and speculations on future research directions. Insect Biochem Mol Biol 30: 617-644

Goodman WG, Granger NA (2005) The juvenile hormones. In "Comprehensive Molecular Insect Science" Ed by LI Gilbert, K latrou, SS Gill, Elsevier Ltd., Oxford, pp 319-408

Granger NA, Ebersohl R, Sparks TC (2000) Pharmacological characterization of dopamine receptors in the corpus allatum of Manduca sexta larvae. Insect Biochem Mol Biol 30: 755-766

Granger NA, Sturgis SL, Ebersohl R, Geng C, Sparks TC (1996) Dopaminergic control of corpora allata activity in the larval tobacco hornworm, Manduca sexta. Arch Insect Biochem Physiol 32: 449-466

Hartfelder K, Emlen DJ (2005) Endocrine control of insect polyphenism. In "Comprehensive Molecular Insect Science" Ed by LI Gilbert, K latrou, SS Gill, Elsevier Ltd., Oxford, pp 651-703

Hawkins PT, Stephens LR (2016) Emerging evidence of signalling roles for PI(3,4)P2 in Class I and II PI3K-regulated pathways. Biochem Soc Trans 44: 307-314

Huang J, Tian L, Peng C, Abdou M, Wen D, Wang Y, et al. (2011) DPP-mediated TGFbeta signaling regulates juvenile hormone biosynthesis by activating the expression of juvenile hormone acid methyltransferase. Development 138: 2283-2291

Ishimaru Y, Tomonari S, Matsuoka Y, Watanabe T, Miyawaki K, Bando T, et al. (2016) TGF-beta signaling in insects regulates metamorphosis via juvenile hormone biosynthesis. Proc Natl Acad Sci USA 113: 5634-5639

Ito T, Sakaki Y (1999) Fluorescent differential display: a fast and reliable method for message display polymerase chain reaction. Methods Enzymol 303: 298-309

Kaneko Y, Hiruma K (2014) Short neuropeptide F (sNPF) is a stagespecific suppressor for juvenile hormone biosynthesis by corpora allata, and a critical factor for the initiation of insect metamorphosis. Dev Biol 393: 312-319

Kaneko Y, Kinjoh T, Kiuchi M, Hiruma K (2011) Stage-specific regulation of juvenile hormone biosynthesis by ecdysteroid in Bombyx mori. Mol Cell Endocrinol 335: 204-210

Kinjoh T, Kaneko Y, Itoyama K, Mita K, Hiruma K, Shinoda T (2007) Control of juvenile hormone biosynthesis in Bombyx mori: cloning of the enzymes in the mevalonate pathway and assessment of their developmental expression in the corpora allata. Insect Biochem Mol Biol 37: 808-818

Koyama T, Obara Y, Iwami M, Sakurai S (2004) Commencement of pupal commitment in late penultimate instar and its hormonal control in wing imaginal discs of the silkworm, Bombyx mori. J Insect Physiol 50: 123-133

Maestro JL, Cobo J, Belles X (2009) Target of rapamycin (TOR) mediates the transduction of nutritional signals into juvenile hormone production. J Biol Chem 284: 5506-5513

Nijhout HF (1994) Insect Hormones, Princeton Univ Pr

Niwa R, Niimi T, Honda N, Yoshiyama M, Itoyama K, Kataoka $\mathrm{H}$, Shinoda T (2008) Juvenile hormone acid O-methyltransferase in Drosophila melanogaster. Insect Biochem Mol Biol 38: 714 720

Raikhel AS, Brown MR, Belles X (2005) Hormonal control of reproductive processes. In "Comprehensive Molecular Insect Science" Ed by LI Gilbert, K latrou, SS Gill, Elsevier Ltd., Oxford, pp 433-491

Riddiford LM (1996) Juvenile hormone: the status of its "status quo" action. Arch Insect Biochem Physiol 32: 271-286

Sakurai S, Niimi S (1997) Development changes in juvenile hormone and juvenile hormone acid titers in the hemolymph and in-vitro juvenile hormone synthesis by corpora allata of the silkworm, Bombyx mori. J Insect Physiol 43: 875-884

Satake S, Kaya M, Sakurai S (1998) Hemolymph ecdysteroid titer and ecdysteroid-dependent developmental events in the lastlarval stadium of the silkworm, Bombyx mori: role of low ecdysteroid titer in larval-pupal metamorphosis and a reappraisal of the head critical period. J Insect Physiol 44: 867-881

Shinoda T, Itoyama K (2003) Juvenile hormone acid methyltransferase: a key regulatory enzyme for insect metamorphosis. Proc Natl Acad Sci USA 100: 11986-11991

Stay B, Tobe SS (2007) The role of allatostatins in juvenile hormone synthesis in insects and crustaceans. Annu Rev Entomol 52: 277-299

Suren-Castillo S, Abrisqueta M, Maestro JL (2012) FoxO inhibits juvenile hormone biosynthesis and vitellogenin production in the German cockroach. Insect Biochem Mol Biol 42: 491-498

Taguchi S, Iwami M, Kiya T (2011) Identification and characterization of a novel nuclear noncoding RNA, Fben-1, which is preferentially expressed in the higher brain center of the female silkworm moth, Bombyx mori. Neurosci Lett 496: 176-180

Tan A, Tanaka H, Tamura T, Shiotsuki T (2005) Precocious metamorphosis in transgenic silkworms overexpressing juvenile hormone esterase. Proc Natl Acad Sci USA 102: 11751-11756

Truman JW, Hiruma K, Allee JP, Macwhinnie SG, Champlin DT, Riddiford LM (2006) Juvenile hormone is required to couple imaginal disc formation with nutrition in insects. Science 312: $1385-1388$

Ueda H, Shinoda T, Hiruma K (2009) Spatial expression of the mevalonate enzymes involved in juvenile hormone biosynthesis in the corpora allata in Bombyx mori. J Insect Physiol 55: 798804

Whisenton LR, Bowen MF, Granger NA, Gilbert LI, Bollenbacher WE (1985) Brain-mediated 20-hydroxyecdysone regulation of juvenile hormone synthesis by the corpora allata of the tobacco hornworm, Manduca sexta. Gen Comp Endocrinol 58: 311-318

Yamanaka N, Yamamoto S, Zitnan D, Watanabe K, Kawada T, Satake H, et al. (2008) Neuropeptide receptor transcriptome reveals unidentified neuroendocrine pathways. PLoS One 3: e3048

(Received April 24, 2017 / Accepted May 17, 2017) 